\title{
Editorial on Dark Matter Theoretical Discernment
}

\section{Manu Mitra}

Department of Electrical Engineering, Alumnus of University of Bridgeport, Bridgeport, United States mmitra@my.bridgeport.edu

\section{INTRODUCTION}

The known material in the universe is called baryonic matter which consists of electrons, protons and neutrons. Dark matter can be made of baryonic or non-baryonic matter. To grip the elements of the universe together, dark matter may makeup eighty percent of its matter (80\%) approximately. Most of the physics experts think that dark matter is made up of non-baryonic matter that have ten to hundred times the mass of a proton and because of their weak correlation with "normal" matter makes it harder to discover. The missing matter can be more challenging to investigate. Massive black holes could also be part of the difference. These hard to detect objects has yet to play very important role. [1]

Few of them below are discussed about dark matter and its phenomenon.

\section{EXPERIMENTAL DATA SHOWS DARK MATTER INTERACTS WITH ORDINARY MATTER}

For the past decade, Yu an expert has led an attempt to bridge particle physics and cosmology by understanding the ways of dark matter's particle properties from astronomical data. Yu and his team discovered a class of dark matter theories with a new dark force that may help us to understand unexpected features seen across a wide range from dwarf galaxies to galaxy clusters.

$\mathrm{Yu}$, a theorist in the department of Physics and Astronomy with his team including Yong Yang co-led a team analyzing and interpreting the latest experimental data showing dark matter interacts with ordinary matter. Yu, also explained that should a dark matter particle hit with PandaX-II's liquefied xenon, the result would be two simultaneous signals: one is proton and other electron. [2]

\section{A Galactic Test Showing Existence of Dark MatTer}

Scientists concentrated on a relationship that is called Radial Acceleration Relation (RAR). In disk galaxies, stars revolve in elliptical orbits around the galactic center. The acceleration that moves them to change direction that is caused by attraction of matter in the galaxy. Radial Acceleration Relation (RAR) that explains the relationship between the acceleration that is caused by only visible matter. This provides some perception into the structure of galaxies and their matter allocation

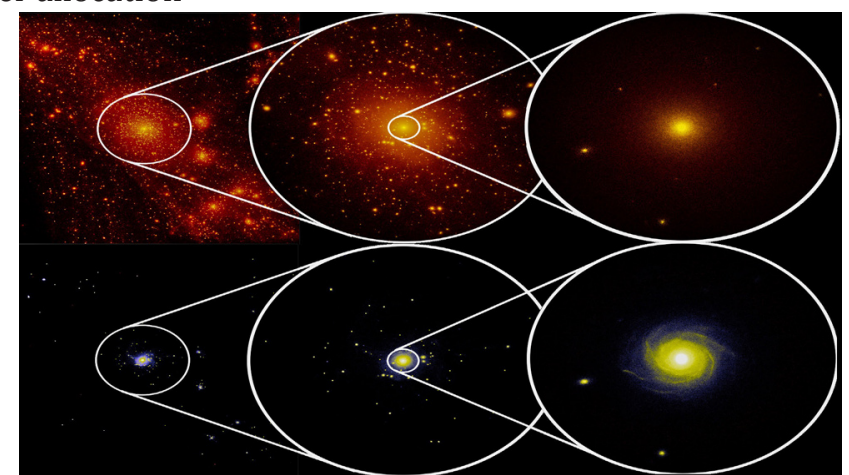

Fig1. Illustrates distribution of dark matter (above) and stars (below). [Reference4] Credit: (c) E. Garaldi, C. Porciani, E. Romano-Díaz/University of Bonn for the ZOMG Kollaboration 
Scientist at University of California, Irvine and University of Bonn used one of the fastest supercomputers workstations that have simulated the matter allocation so called satellite "dwarf" galaxies. These are mini galaxies that orbit larger galaxies like Milky Way. [3]

\section{Dark Matter Goes Missing in Galaxy}

Galaxies and dark matter typically go together like peanut butter and jelly. Normally, you don't find one without another. Physicist were surprised when they discovered that a galaxy that is most of its dark matter. A substance that is invisible, dark matter is the underlying platform on which galaxies are built. It's like a glue that grips the visible matter in the galaxies, stars and gas together.

The rare galaxy called NGC 1052-DF2 contains at most $1 / 400^{\text {th }}$ the quantity of dark matter that astronomers expected. This galaxy is as massive as Milky Way but escaped the attention of scientist because it contains only $1 / 200^{\text {th }}$ the number of stars. Because of the object's huge size and faint appearance, expert astronomers classify NGC 1052-DF2 as an ultra-diffuse galaxy. [5, 6]

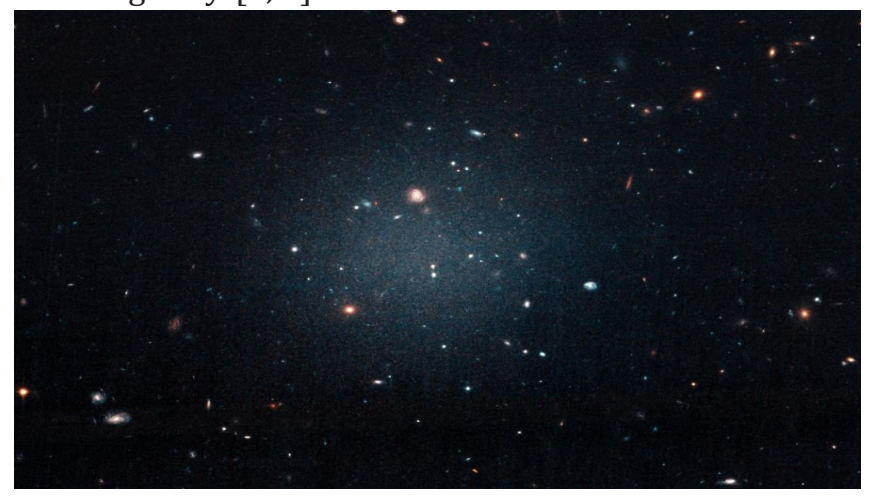

Fig2. Illustrates fuzzy looking galaxy broadly distributed that astronomers call "see-through" galaxy because they can see clearly distant galaxies. All these pale in comparison is the strange aspect of this galaxy; NGC 1052-DF2 is missing most if not all, of its dark matter. [Reference 6]Credit: NASA, ESA, and P. van Dokkum (Yale University)

\section{Unlocking SECRETS OF DARK MATTER}

Astrophysicist Cappelluti's study on dark matter was examined that an interesting light source that was picked up by four various telescopes each pointing in divergent direction in the sky. The source of light is obscure and not recognizable to astrophysicists and caused a quite an activity in the world of astrophysics. Bulbul also found that emission line while examining clusters of galaxies in the year 2014. As per Esra Bulbul, an expert astrophysicist at Harvard Smithsonian Center for Astrophysics; about ninety five percent of the mass in the universe is consist of material that is unknown and invisible to expert scientists, that is dark matter.

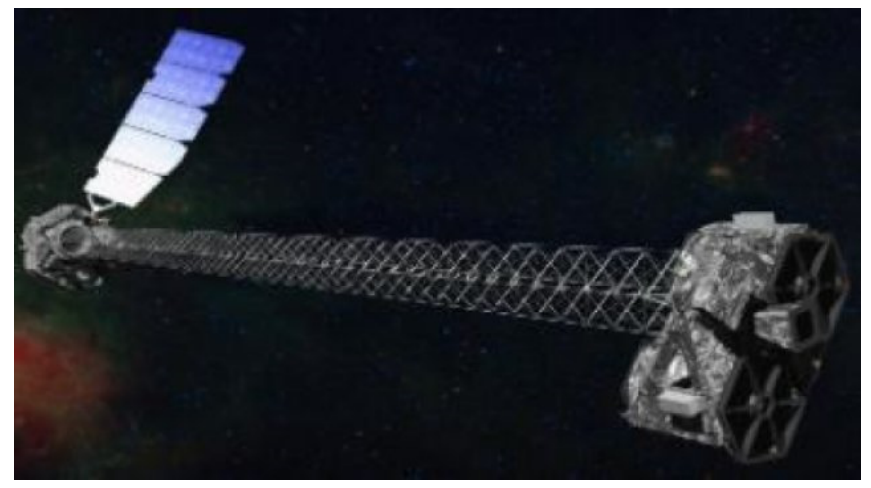

Fig3. Illustrates NASA's NuSTAR telescope. [Reference 8] Image Credit: NASA 
"We used special telescopes to attain X-ray light in the sky, while looking at X-rays, and observed an unexpected feature which was captured spectrum of light, which is not created by any known atomic emission. This emission line called the 3.5 kiloelectron volt (keV). By analysis of this emission line; created by the decay of dark matter" was confirmed by astrophysicist Cappelluiti. [7, 8]

\section{Hunting FOR DARK MATTER}

Astrophysicists from University of Surrey and University of Edinburgh designed a new method to measure the amount of dark matter for tiny "dwarf" galaxies. They created a new method to calculate the inner dark matter density from dwarf galaxies, even if there is absence of gas and left with few stars. The most important part of the method is to make use of one or more dense star clusters orbiting nearby to the center of the dwarf.

"We designed a new tool to discover the nature of dark matter and investigation results are great. Eridanus II one of the smallest galaxies known to us, has few dark matter in its center than anticipated. If alike results are found for sample of galaxies, this can have wide range implications for dark mater investigation" was told by Dr. Filippo Contenta from University of Surrey. $[9,10]$

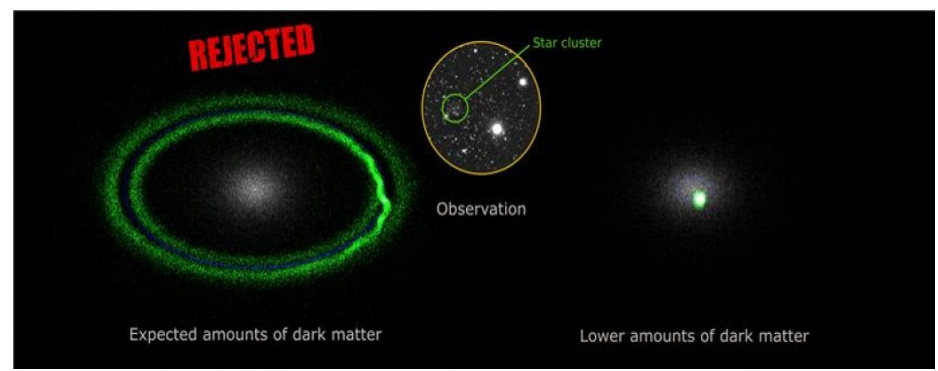

Fig4. Dark matter makes up most of the mass of the Universe, yet it remains elusive.[Reference 10] Image Credit: Observational image taken from Crnojevi et al. 2016; composite image produced by DrMaxime Delorme (University of Surrey)

\section{ACKNOWLEDGMENT}

Author would like to thank Prof. Navarun Gupta, Prof. Hassan Bajwa, Prof. Linfeng Zhang and Prof. Hmurcik for their academic support. Author also thanks anonymous reviewers for their comments.

\section{REFERENCES}

1. Redd, N. T. (2017, November 14). What is Dark Matter? Retrieved from https://www.space.com/20930dark-matterhtml

2. Xiangxiang Ren et al. Constraining Dark Matter Models with a Light Mediator at the PandaX-II Experiment, Physical Review Letters (2018). DOI: 10.1103/PhysRevLett.121.021304

3. Enrico Garaldi et al, Radial Acceleration Relation of $\Lambda$ CDM Satellite Galaxies, Physical Review Letters (2018). DOI: 10.1103/PhysRevLett.120.261301

4. Enrico Garaldi et al. (2018). The distribution of dark matter (above) and stars (below) [photograph]. Retrieved from https://phys.org/news/2018-06-galactic-dark.html

5. Pieter van Dokkum, ShanyDanieli, Yotam Cohen, Allison Merritt, Aaron J. Romanowsky, Roberto Abraham, Jean Brodie, Charlie Conroy, Deborah Lokhorst, LamiyaMowla, Ewan O'Sullivan \&Jielai Zhang. A galaxy lacking dark matter. Nature, 2018 DOI: 10.1038/nature25676

6. NASA/Goddard Space Flight Center. (2018, March 28). Dark matter goes missing in oddball galaxy. ScienceDaily. Retrieved July 18, 2018 from www.sciencedaily.com/releases/2018/03/ 180328130724.htm 
Editorial on Dark Matter Theoretical Discernment

7. Nico Cappelluti, Esra Bulbul, Adam Foster, Priyamvada Natarajan, Megan C. Urry, Mark W. Bautz, Francesca Civano, Eric Miller, Randall K. Smith. Searching for the $3.5 \mathrm{keV}$ Line in the Deep Fields with Chandra: The 10 Ms Observations. The Astrophysical Journal, 2018; 854 (2): 179 DOI: 10.3847/1538-4357/aaaa68

8. University of Miami. (2018, April 16). An astrophysicist is unlocking the secrets to dark matter. ScienceDaily. Retrieved July 18, 2018 from www.sciencedaily.com/releases/2018/04/180416121533.htm

9. Filippo Contenta, Eduardo Balbinot, James A Petts, Justin I Read, Mark Gieles, Michelle L M Collins, Jorge Peñarrubia, Maxime Delorme, AlessiaGualandris. Probing dark matter with star clusters: a dark matter core in the ultra-faint dwarf Eridanus II. Monthly Notices of the Royal Astronomical Society, 2018; 476 (3): 3124 DOI: $10.1093 / \mathrm{mnras} / \mathrm{sty} 424$

10. University of Surrey. (2018, April 6). Hunting for dark matter in the smallest galaxies in the Universe. ScienceDaily. Retrieved July 18, 2018 from www.sciencedaily.com/releases/2018/04/ 180406091758.htm

Citation: Manu Mitra, “Editorial on Dark Matter Theoretical Discernment". American Research Journal of Electronics and Communication Engineering; vol. 1, no. 1; pp: 28-31.

Copyright (c) Manu Mitra. This is an open access article distributed under the Creative Commons Attribution License, which permits unrestricted use, distribution, and reproduction in any medium, provided the original work is properly cited. 